Approved for public release; distribution is unlimited.

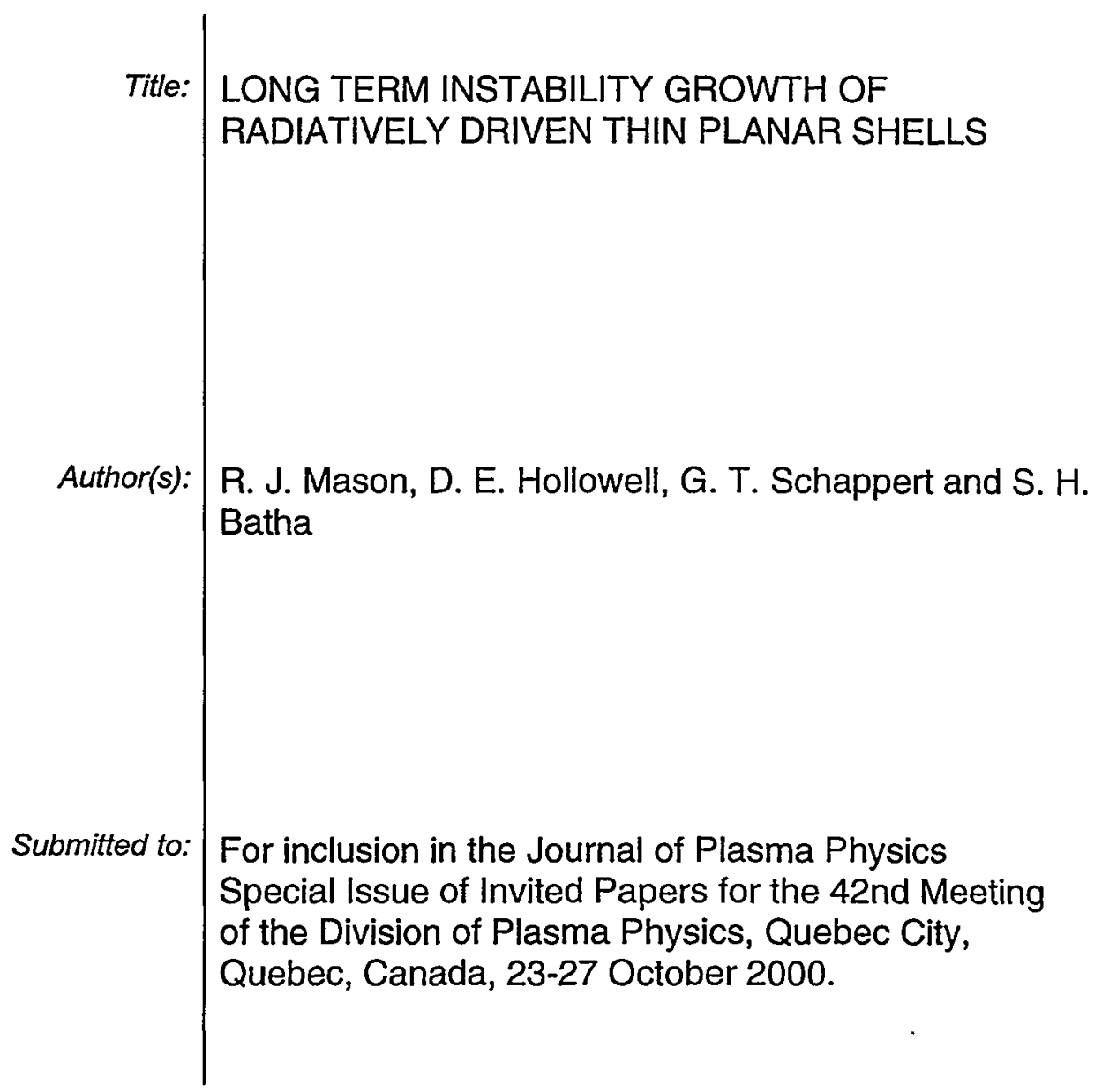

\section{Los Alamos}

Los Alamos National Laboratory, an affirmative action/equal opportunity employer, is operated by the University of Califomia for the U.S. Department of Energy under contract W-7405-ENG-36. By acceptance of this article, the publisher recognizes that the U.S. Government retains a nonexclusive, royalty-free license to publish or reproduce the published form of this contribution, or to allow others to do so, for U.S. Government purposes. Los Alamos National Laboratory requests that the publisher identify this article as work performed under the auspices of the U.S. Department of Energy. Los Alamos National Laboratory strongly supports academic freedom and a researcher's right to publish; as an institution, however, the Laboratory does not endorse the viewpoint of a publication or guarantee its technical correctness. 


\section{DISCLAIMER}

This report was prepared as an account of work sponsored by an agency of the United States Government. Neither the United States Government nor any agency thereof, nor any-of their employees, make any warranty, express or implied, or assumes any legal liability or responsibility for the accuracy, completeness, or usefulness of any information, apparatus, product, or process disclosed, or represents that its use would not infringe privately owned rights. Reference herein to any specific commercial product, process, or service by trade name, trademark, manufacturer, or otherwise does not necessarily constitute or imply its endorsement, recommendation, or favoring by the United States Government or any agency thereof. The views and opinions of authors expressed herein do not necessarily state or reflect those of the United States Government or any agency thereof. 


\section{DISCLAIMER}

Portions of this document may be illegible in electronic image products. Images are produced from the best available original document. 


\title{
Long Term Instability Growth of Radiatively Driven Thin Planar Shells
}

\author{
R. J. Mason, D. E. Hollowell, G. T. Schappert and S. H. Batha \\ Los Alamos National Laboratory
}

We study Rayleigh-Taylor instability of radiatively driven thin copper foils under pure ablation, as well as with beryllium tampers to provide additional pressure drive. Modeling was done with the RAGE adaptive mesh refinement code of experiments done on the NOVA and OMEGA lasers. The copper foils were typically $11.5 \mu \mathrm{m}$ thick with $0.45 \mu \mathrm{m}$ amplitude, $45 \mu \mathrm{m}$ wavelength cosine surface perturbations. The beryllium layer was $5 \mu \mathrm{m}$ thick. The drive was a "P26"-like laser pulse delivering a peak 160-185 eV radiation temperatures. Good agreement between experiment and simulation has been obtained out to $4.5 \mathrm{~ns}$. Mechanisms for late time agreement are discussed.

\section{INTRODUCTION}

Whenever a lower density fluid is made to push on a higher density fluid, there is a growth of perturbations on the surface at the density interface due to Rayleigh-Taylor (R-T) instability ${ }^{1,2}$. The radiation in laser driven hohlraums can be used to drive such instability ${ }^{3}$. With currently available laser energies, typically $20 \mathrm{~kJ}$, this instability, at the high-density interfaces provided by metals in air, can only be studied in the motion of thin foils. Radiative ablation drives lowdensity ejected gas that accelerates such foils ${ }^{4}$. In recent years such ablative Rayleigh-Taylor instability has been seduloulsy scrutinized for ICF application ${ }^{5,6}$. Low density, plastic foam buffers have been shown to help stabilize foils directly driven by perturbed laser beams ${ }^{7,8}$.

Conversely here, we are interested in destabilizing effects from the additional push that can come from the presence of a lower density tamper layer ${ }^{9}$ on the drive side of the foils abutting the initial surface perturbations. The extra material pressure from such tampers is shown to increase the acceleration of the foils, and hasten their breakup from R-T instability. We have conducted early experiments at the Lawrence Livermore National Laboratory on NOVA ${ }^{10}$, and most recently at the Rochester Laboratory for Laser Energetics on OMEGA ${ }^{11}$. Both sets of experiments displayed this increased instability. Detailed modeling with and LASNEX ${ }^{12}$ and with Los Alamos National Laboratory's RAGE ${ }^{13}$ adaptive mesh refinement (AMR) code has corroborated these results.

In what follows, we first describe the laser targets used and the experiments measuring target instability. We discuss one-dimensional simulations, showing advancing radiation profiles, and the resultant driven dynamics of thin copper foils. We next examine the effects of a thin beryllium buffer layer that is used at least to filter the radiative drive. We show calculated increased net drive, when this layer abuts the foils. We then discuss the two-dimensional calculations used to track R-T instability growth. Electron thermal conductivity is found to provide important density profile smoothing. Mesh refinement tracks evolving density details. Finally, in comparing experiment and simulation, we find generally good agreement during an early non-linear regime, during which R-T spikes grow to 20 times the initial disturbance 
amplitude. We conclude with a survey of various adjustments that can be applied to the data to produce a good match to our numerical simulations at later times.

\section{EXPERIMENTAL BACKGROUND}

Our experiments have focused on shell targets that are substantially thinner than the perturbation wavelength. We have studied the contrasting dynamics obtained with just simple ablative drive, and then with the addition of material pressure drive. We anticipated and observed greater foil acceleration, and faster Rayleigh-Taylor (R-T) growth with the extra material pressure. We have focused on high $\mathrm{Z}$ shells to see $\mathrm{R}-\mathrm{T}$ effects associated with high density in the hydrodynamics and high opacity in the radiation transport. Available laser energy limited us to copper. Figure 1 shows schematics of our two target classes. We used $5 \mu \mathrm{m}$ of beryllium in each case to provide a constant filtering of the light reaching the copper. For the pure ablative drive the beryllium was set as a flat slab spaced $200 \mu \mathrm{m}$ beyond the $11.5 \mu \mathrm{m}$ copper foil. For the pressure drive the beryllium was contoured onto the perturbed surface of our foils. For our present studies the initial perturbations of the copper surfaces were a single mode 0.45 $\mu \mathrm{m}$ in amplitude with a $45 \mu \mathrm{m}$ wavelength.

At Rochester, $1.4 \mathrm{~mm}$ diameter tetrahedral hohlraums were irradiated by 45 beams of OMEGA, each beam typically delivering $400 \mathrm{~J}$ of $3 \omega, 0.35 \mu \mathrm{m}$ light for a total of $18 \mathrm{~kJ}$. This produced a peak drive radiation temperature of $\sim 170 \mathrm{eV}$. Twelve additional beams delivering up to $4.4 \mathrm{~kJ}$ were reserved for face-on radiography. The beams impinged on a $0.5 \mathrm{mil}$ iron backlighter, producing $6.7 \mathrm{keV}$ temperature radiation for target imaging in a framing camera looking normal to the target, as shown in Fig. 2. Such high photon energy was needed for the penetration of copper spikes growing to more than $20 \mu \mathrm{m}$ in length from the R-T instability. In all cases, the copper foil in our R-T packages was positioned with the spacers some $200 \mu \mathrm{m}$ off the hohlraums wall.

A typical radiograph from the backlighter appears in Fig. 3, which shows at least nine 45 $\mu \mathrm{m}$ wavelengths of the growing perturbation. In analyzing this image, we first removed the characteristics of the pure backlighter beam (the image with no package). We averaged the data recorded on a strip over three 60 ps intervals to minimize fluctuations. We also applied a Wiener filter for noise mitigation. The result was Fourier analyzed (left to right below), and corrected for the modulation transfer function of the camera. We assumed a constant opacity through the copper, measured from the brightness of our images an effective integrated $\rho R$ through the copper, and by dividing away a cold copper density of $8.93 \mathrm{~g} / \mathrm{cm}^{3}$, we determined an effective length for the perturbations. Typical exposures, as in figure 3 , show fine scale grooves in the highest exposure points, corresponding to possible spikes in the center of the growing R-T bubbles.

The tetrahedral hohlraums on the OMEGA laser provide a spherical cavity matching the natural OMEGA symmetry. They can be driven with up to $24.5 \mathrm{~kJ}$ in pulse shape " 26 " - NOVA parlance. Our earlier NOVA experiment had used cylindrical hohlraums at comparable energies with both the backlighter and target packaged mounted in opposition at mid-axis. Our most reliable data has come from OMEGA, however, on which we concentrate here. With the laser history corresponding to $18 \mathrm{~kJ}$ of deposition in a NOVA-like "P26" pulse, Fig. 4(a), we typically recorded a radiative drive temperature history, Fig. $4(\mathrm{~b})$, peaking at about $160-185 \mathrm{eV}$. Here, $\mathrm{t}=0$ is somewhat arbitrary, but the drive is above $100 \mathrm{eV}$ for about $2 \mathrm{~ns}$. We idealized this as the 
radiation temperature $T_{r}$ boundary condition of Fig. $4(c)$ for use in our LASNEX and RAGE calculations.

\section{SIMULATIONS}

\section{A. 1D LASNEX calculations}

Early scoping calculations for this experiment were first performed with LASNEX. For the bare copper foils these calculations showed, for example, the results of Fig. 5. For a peak radiation temperature of $160 \mathrm{eV}$ the "p26" pulse gives a $40 \mathrm{eV}$ value in the drive region at $1 \mathrm{ns,}$ rises to $85 \mathrm{eV}$ by $4 \mathrm{~ns}$, is near its peak at $2 \mathrm{~ns}$, and then starts to push the foil to the left in the figure. The foil resides initially at $x=0$. By $6 \mathrm{~ns}$ its peak density point has moved to $x \approx-170$ $\mu \mathrm{m}$. The peak $\mathrm{Cu}$ density goes to $16 \mathrm{~g} / \mathrm{cm}^{3}$ at $2 \mathrm{~ns}$, drops back to $7.5 \mathrm{~g} / \mathrm{cm}^{3}$ by $3 \mathrm{~ns}$, and to only 4 $\mathrm{g} / \mathrm{cm}^{3}$ by $6 \mathrm{~ns}$. At this late time the drive just ahead of the density peak is down to only $40 \mathrm{eV}$.

In one-dimension we calculated mass trajectories that matched side-on radiography measurements conducted at NOVA. For OMEGA typical zonal $\mathrm{x}-\mathrm{t}$ plots for bare $\mathrm{Cu}$ foils are given in Fig. 6. The radiation is first absorbed by the Be layer, which here serves only as a filter. This filter initially resides $200 \mu \mathrm{m}$ in front of the foil, separated from the foil by the spacers of Fig. 1(b). It explodes, with its outer regions moving toward the driving radiation and reaching $600 \mu \mathrm{m}$ by $1.9 \mathrm{~ns}$. The filter's inner mass heads toward the copper foil, striking its expanding outer regions by about 700 ps. After 1 ns roughly all of the filter mass is blowing toward the driver. The ablating $\mathrm{Cu}$ mass reaches $\sim 400 \mu \mathrm{m}$ by $6 \mathrm{~ns}$, while copper zones clustering near the density peak are pushed back to $\mathrm{x} \approx-350 \mu \mathrm{m}$ by $10 \mathrm{~ns}$.

If the filter is placed at $200 \mu \mathrm{m}$ or beyond, its presence has little hydrodynamic effect on the peak density trajectory for the $\mathrm{Cu}$ foil. When, for example, we initiated it at $2000 \mu \mathrm{m}$, the resultant density trajectory was the same. The filter does, however, weaken the drive, retarding its arrival at the copper. We can see this from Fig. 7(a). At $1 \mathrm{~ns}$, when the drive is at $100 \mathrm{eV}$ in the hohlraum, it is still only $60 \mathrm{eV}$ adjacent to the foil. However, by $1.3 \mathrm{~ns}$ the radiation temperature profile $T_{r}$ is nearly flat across the filter. Trajectories of the foil peak densities show that with remote Be filtering the foils move most slowly, due to the delay in the drive at the foil. In unfiltered light the copper foils move about $25 \mu \mathrm{m}$ further by $6 \mathrm{~ns}$. This is shown in Fig. 7(b). Finally, with the $\mathrm{Be}$ attached to the foil to provide additional pressure as a buffer layer, the copper moves the furthest distance - to a point $\sim 50 \mu \mathrm{m}$ ahead of the bare filtered foil by $6 \mathrm{~ns}$. We retained the beryllium filter in the bare copper experiments to assure a similar drive on the copper with and without buffering.

More general parameter studies confirmed that, optimally for pressure acceleration, the beryllium layer should initially touch the copper. The beryllium layer is largely penetrated by the radiation at $1 \mathrm{~ns}$. Figure 8 compares at $1 \mathrm{~ns}$ the density $\rho$ and radiative temperature, $T_{r}$, profiles for a remote Be layer versus an attached Be buffer layer. In each case, the $T_{r}$ peaks at $65 \mathrm{eV}$ in the $\mathrm{Cu}$, but the copper density is shocked only 1.3-fold in the bare case. In the buffered case, the beryllium layer is seen still abutting the foil, and the shock exhibits a 3.1-fold increase in density. Additional studies, in which the buffering beryllium mass was kept constant, but with its initial density artificially reduced, showed that the greatest drive came at natural, solid density. When, for example, the beryllium was treated computationally as a $0.1 \mathrm{~g} / \mathrm{cm}^{3}$ density foam, the peak 
shock density in the copper at 2 ns was $25 \%$ lower than with a solid Be buffer, indicative of weaker shocking of the copper by the same drive. Consequently, we avoided the use of foams in our experiments.

\section{B. 2D RAGE results}

For two-dimensional calculations we found that the LASNEX mesh tangled seriously, just as important non-linear features in the R-T growth were emerging; therefore, we turned to RAGE. This Eulerian model treats the interfaces between the copper, beryllium and background void and regions of steep density and temperature variation with adaptive mesh refinement. The largest cells in our calculations -- generally in the void -- were $1.125 \mu \mathrm{m}$ square. Our smallest cells - mostly those near the interfaces -- were three levels higher in refinement, i.e. $0.1406 \mu \mathrm{m}$ on a side. This gave us lateral 320 points to resolve the initial $45 \mu \mathrm{m}$ perturbation, and 8.6 cells to resolve the $9 \mu \mathrm{m}$ peak-to-valley amplitude perturbations. To avoid singularities in the Eulerian calculations, the void was actually treated as a low density, $10^{-3} \mathrm{~g} / \mathrm{cm}^{3}$, air. Figure 9 below shows a typical mesh setup as the calculations start at $\mathrm{t}=0$.

For all the calculations reported here the radiative drive was supplied as a temperature boundary condition on the right at the $1200 \mu \mathrm{m}$ position along the $\mathrm{x}$-axis. In figure 5 the right (drive) side of the beryllium tamper was flat while the left side had a cosine profile, making the beryllium $6 \mu \mathrm{m}$ thick in the center. More generally, the buffer layer had a cosine profile on both sides, being an equal $5 \mu \mathrm{m}$ thick at all the vertical (y) positions. We found no noteworthy dependence on the flatness of the outer tamper surface.

Our initial runs were made with radiation transport "on," but electron thermal conduction "off." The rapid development of fine scale density filaments or "filigree" plagued the results of these runs. This appeared on the drive side of the copper or beryllium. The source of the effect is purely hydrodynamic and persists when the radiation transport is suppressed and the foils are accelerated by an equivalent push from high-pressure air.

The RAGE mesh has difficulty representing a smooth cosine surface profile on the background Cartesian mesh. The steep density gradients at the copper interface with air or beryllium introduces stair-stepped density contours following the edges of the square mesh elements. The density steps serve as short wavelength seeds for instability. Even with AMR, the smallest cells must be crossed diagonally by the surface interface, with mass fractions apportioned on either side. In a mixed cell the smallest fraction of copper, for example, permitted is $\left(1 / 2^{n}\right)^{2}$. We started with $n=2$ and found some reduction in the short wavelength seeding, when we increased $n$ to 8 . The seeding could be further reduced when we terraced the interface between the copper and the air or beryllium by introducing a one row of cells set at one third solid copper density. The initial density drop then occurred over three cells rather than two. This is a somewhat cumbersome manual procedure, however. We achieved the greatest smoothing, as shown below in figure 10 , by simply turning on the electron thermal transport. The figure compares the density profiles at $4 \mathrm{~ns}$ with thermal conductivity $\mathrm{K}_{e}$ "on" and "off." The white parabolic curve is a shock passing through the copper. In our experiment thermal conductivity delivers heat to the micron size filaments, and fire polishes them away.

When we looked at $5.6 \mathrm{~ns}$ at a sequence of bare filtered foils under the same "P26" drive, but with initial thickness ranging from 15 down to $10 \mu \mathrm{m}$, we found, properly, that the thinner, fastest moving foils developed the longest spikes, as in Fig. 11, helping us to "tune" our 
experiments. We see that by this $5.6 \mathrm{~ns}$ the $11.5 \mu \mathrm{m}$ foil has developed spikes $\sim 30 \mu \mathrm{m}$ long. We shall see that this presents a problem for our experimental diagnostics.

Comparing our calculations employing electron thermal conductivity for the buffered and bare foil accelerations, we find that the pressure from the beryllium buffer does, indeed, provide an extra push to the copper foil. The two foils start at the $x=600 \mu \mathrm{m}$ position in figure 12(a) and (b). The figure illustrates that in response to a $160 \mathrm{eV}$ peak drive temperature the tamping drives the center of the copper foil $50 \mu \mathrm{m}$ further to the left by $4 \mathrm{~ns}$. The buffered foil develops a strong central filament in the R-T bubble, and side fingers indicative of increased $2^{\text {nd }}$ and fourth harmonic growth. Both targets show the development of spikes adjacent to the walls exceeding $30 \mu \mathrm{m}$ in length. Figures 12 (c) and (d) show the modal growth with time of the disturbed amplitude, as calculated with RAGE. We post-processed the code output to obtain values of $\rho R$ $=\int \rho(\mathrm{x}, \mathrm{y}) \mathrm{dx}$ at 40 lateral positions $\mathrm{y}$. The $\rho \mathrm{R}$ contributions from any initial beryllium and the vacuum (low density air) were subtracted away. The result was then divided by the initial copper density to determine a foil thickness at the various lateral positions, $\tau(y)$. This was Fourier analyzed to yield (c) and (d). At $4 \mathrm{~ns}$ the first four modal amplitudes in the buffered case (c) are $6.6,4.0,2.4$ and $1.0 \mu \mathrm{m}$, summing to $14.0 \mu \mathrm{m}$. Higher order modes (to about the $10^{\text {th }}$ ) sum to another $6 \mu \mathrm{m}$ and are needed to produce the large spikes, top and bottom.

\section{COMPARED EXPERIMENTS AND CALCULATIONS}

We compare our data from the OMEGA experiments with our RAGE predictions in figure 13. Each data point is the average of 4 measurements recorded on a film strip at 60 ps separation to minimize noise, as discussed with Fig. 3. The predicted first order perturbations (determined as above) start at $0.45 \mu \mathrm{m}$ and grow beyond $6 \mu \mathrm{m}$ in amplitude (effective length) by $6 \mathrm{~ns}$. Our calculations for the first and second harmonic growth are in good agreement with the data until about $4.5 \mathrm{~ns}$ for the bare copper, and $4.0 \mathrm{~ns}$ for the buffered copper foil.

As a possible explanation for this discrepancy, we noted that our observation of the backlighter was not truly face-on but was instead, typically, at about $4^{\circ}$ to the side. This would lead to a reduced $\rho R$ measurement, as we looked slightly across a spike instead of straight down its extent. Simulations of this effect in buffered foils showed, however, that as much as a $20^{\circ}$ observation angle would be needed to reduce the calculated first harmonic amplitude from $\sim 9 \mu \mathrm{m}$ to the observed $5 \mu \mathrm{m}$ at $5.5 \mathrm{~ns}$.

Second, it is likely that the effective temperature driving the foils was lower than that measured in the hohlraums at late time, by virtue of energy loss at the edges of the foil package as it lifted off the hohlraum wall. The spacers (Fig. 1) holding the package allowed for some leakage even at $t=0$. We crudely modeled this effect by truncating the radiative drive after 2.2 ns, when the copper was calculated to move off the hohlraum through its initial $11 \mu \mathrm{m}$ thickness. We found that this could bring the buffered data and simulation into better accord for both the first and second harmonics. However, the bare data is better matched without truncation, possibly because it separates from the hohlraum more slowly.

Finally and most significantly, we observed at OMEGA that before saturation our $6.7 \mathrm{keV}$ iron backlighter illumination can only penetrate about 15 to $20 \mu \mathrm{m}$ of copper. With a copper cold opacity of about $\mathrm{K}_{\mathrm{r}}=80 \mathrm{~cm}^{2} / \mathrm{g}$ (appropriate at $\sim 160 \mathrm{eV}$ material temperatures for the 6.7 $\mathrm{keV}$ iron irradiation), and the density, $\rho=8.93$, the backlighter intensity is reduced to $15 \%$ of its initial value, upon penetrating $26 \mu \mathrm{m}$ of the copper. Our backlighter detector is incapable of 
discrimination at lower intensities. Generally, considering the backlighter decay through the full foil at various transverse positions, i.e. $\mathrm{I}=\mathrm{I}_{0} \exp \left[-\rho \mathrm{K}_{\mathrm{r}} \tau(\mathrm{y})\right]$, one will measure an effective foil thickness $\tau_{e}(\mathrm{y})=-\left(1 / \rho \mathrm{K}_{\mathrm{r}}\right) \log \left\{\exp \left[-\rho \mathrm{K}_{\mathrm{r}} \tau(\mathrm{y})\right]+\Delta \mathrm{I} / \mathrm{I}_{\mathrm{o}}\right\}$, with $\Delta \mathrm{I}=\mathrm{I}-\mathrm{I}_{\mathrm{o}}$, where the $\Delta \mathrm{I} / \mathrm{lo}$ has been added to represent uncertainty at the lowest intensities. Then, should an instability spike become extremely large $\tau \rightarrow \infty$, one will measure $\tau_{\mathrm{e}}$ (in microns) $\rightarrow-(1 / \mathrm{pK} \mathrm{r}) \log \left\{\Delta \mathrm{I} / \mathrm{I}_{0}\right\}=-14.0 \log \{\Delta \mathrm{I} / \mathrm{I}\}$ as an upper limit. Should we be able to measure down to level $\Delta \mathrm{I} / \mathrm{I}_{0}=0.1$, i.e. $10 \%$, of the initial intensity, we would be able to see spikes of maximal length, $\tau_{\mathrm{e}}=31.4 \mu \mathrm{m}$.

Figure 14 shows the RAGE predictions adjusted for such possible minimal sensitivities $\Delta \mathrm{J} / \mathrm{I}$ ranging from $20 \%$ down to $10 \%$. Here, we Fourier analyzed the effective thickness $\tau_{e}$, extracted the first and second harmonics, and plotted them with the corresponding experimental data. We see that the first harmonic data is best matched for a minimum sensitivity between 15 and $20 \%$. The second harmonic is still only matched out to $4.5 \mathrm{~ns}$.

As an alternative, we simply put a $20 \mu \mathrm{m}$ ceiling on the total $\tau(\mathrm{y})$ derived from our RAGE calculations for a $160 \mathrm{eV}$ drive temperature maximum, and Fourier analyzed the result. The buffered case with film-strip averaged (as for Figs. 13 and 14) data is shown in Fig. 15(a). For all measured times this yielded good agreement of the our first harmonic calculations with data for the bare and the buffered foils. The calculated second harmonic is also in good agreement with the bare target data, but still too high for the buffered case. For Fig. 15(b) we used the same 20 $\mu \mathrm{m}$ ceiling on $\tau$, and averaged the same data into time bins $60 \mathrm{ps}$ wide, since different film strips could, in fact, contain data from times overlapping the same time on neighboring strips. This gives a much more detailed agreement with the data, for the first harmonic, except for one outlier for 3.9 ns. Lastly, in Fig. 15(c) we compare strip-averaged data from the buffered targets to our calculated results with the $20 \mu \mathrm{m}$ ceiling a higher $185 \mathrm{eV}$ peak drive temperature. Here, the calculated modal growth is faster, so that the ceiling on total amplitudes is encountered sooner, resulting in less agreement with the first harmonic data and better second harmonic agreement beyond $4 \mathrm{~ns}$.

\section{CONCLUSION}

LASNEX modeling has provided good picture of the effects of radiative filtering by our beryllium layer. Two-dimensional modeling with RAGE provided smooth results in accord with measurements through the use of contoured initial data and the inclusion of electron thermal conductivity. In both experiments and simulation, due to the additional material backpressure, the Be-buffered foils showed enhanced acceleration and increased instability over bare ablating foils. In our simulations of modal growth, amplitude reductions from angular backlighting, prove to be minimal, and the effects of moderate variations in the peak hohlraum temperature are inconclusive. Finally, the measured spike growth amplitudes appear to saturate due to rangingout of the probing backlighter illumination. Thus, good numerical agreement with our measurements is achieved assuming saturation of the backlighter beyond $20-26 \mu \mathrm{m}$ of $\mathrm{Cu}$, corresponding to strongly diminished instrument sensitivity at $20-15 \%$ of the initial backlighter intensity. 


\section{REFERENCES}

1. G. Taylor, Proc. Royal Soc. (London) A 201, 192 (1950).

2. S. Chandrasekar, "Hydrodynamic and Hydromagnetic Stability," Dover Press, NY 1961.

3. E. G. Gamaly, "Hydrodynamic Instability of Target Implosion in ICF," in Nuclear Fusion by Inertial Confinement, Eds. G. Velarde, Y. Ronen, and J. M. Marinez-Val, CRC Press, Boca Raton, 1993.

4. 2. K. Budil, M. Edwards, B. Lasinski, B. Remington, L. Suter, A. Wan, P. Stry, Bull. Am. Phys. Soc. 44(7), 57 (1999).

5. B. A. Remington et al., Phys. Plasmas 2, 241 (1995).

6. H. Takabe et al., Plasma Phys. Controlled Fusion 41, A75 (2000).

7. R. J. Mason, R. A. Kopp, H. X. Vu, D. C. Wilson, S. R. Goldman, R. G. Watt, M. Dunne, and O. Willi, Phys. Plasmas 5, 211 (1998).

8. R. G. Watt, R. J. Mason, J. Duke, C. J. Fontes, P. L. Gobby, R. V. Hollis, R. A. Kopp, D. C. Wilson, C. P. Verdon, T. R. Boehly, J. P. Knauer, D. D Meyerhofer, V. Smalyuk, R. P. Town, A. Iwase, and O. Will, Phys. Rev. Lett. 81, 4644 (1998).

9. R. Mason, D. Hollowell, G. Schappert, and S. Batha, Bull. Am. Phys. Soc., 44(7), 57 (1999).

10. J. D. Lindl, Phys. Plasmas 2, 3933 (1995).

11. J. M. Soures, Phys. Plasmas 3, 2108 (1996).

12. G. B. Zimmerman and W. L. Kruer, Comments Plasma Phys. Controlled Thermonucl. Fusion 2, 51 (1975). 
13. R. M. Baltrusaitis, M. L. Gittings, R. P. Weaver, R. F. Benjamin, and J. M. Budzinski, Phys. Fluids 8, 2471 (1996). 


\section{Figure Captions}

1. Buffered (top), and bare filtered targets

2. Schematic of OMEGA experiment

3. X-ray backlighter image and corresponding exposure of the growing perturbations. Finestructure is evident in spike peaks.

4. (a) Laser pulse shape, (b) resultant radiative drive history, (c) LASNEX and RAGE input radiation temperature, $T_{\mathrm{r}}$ history.

5. One-dimensional evolution of filtered bare copper foils. (a) Radiation temperature, $T_{r}$ history, (b) Evolving densities.

6. (a) $\mathrm{x}$-t history of the Lagrangian zone boundaries; radiation delivered from above.

7. (a) Demonstrated delay in the radiative drive near the foil due to the presence of a $\mathrm{Be}$ filter. (b) Trajectories of the peak density points for i) filtered bare, ii) unfiltered bare and iii) buffered $\mathrm{Cu}$ foils. The filtered bare foil is the slowest.

8. Density and $T_{r}$ profiles at $1 \mathrm{~ns}$ for: a) the bare filtered $\mathrm{Cu}$ foil, and $\mathrm{b}$ ) the Be-buffered foil -- with buffering a layer of $\mathrm{Be}$ is still "leaning" up against the $\mathrm{Cu}$ to give it the additional push which leads to the stronger shock.

9. Initial RAGE mesh showing fine resolution near material interfaces.

10. Electron thermal conduction dramatically reduces the fine scale growth.

11. A sequence of accelerated bare foils varied initial thickness.

12. The buffered foil shows greater acceleration at $4 \mathrm{~ns}$ and the development of a strong central density feature.

13. Perturbation growth in the buffered foil is greater. Agreement of the RAGE calculations with data is good at early times.

14. RAGE predictions for modal growth, assuming instrument insensitivity below $\Delta \mathrm{J} / \mathrm{I}=0.2$.

15. Comparison of: (a) strip-averaged data, and (b) time-binned data to calculations, and (c) the strip-averaged comparison for a higher, $185 \mathrm{eV}$ peak hohlraum temperature. 


\section{$200 \mu \mathrm{m}$ spacer}

$5 \mu \mathrm{m} \mathrm{Be}$

$11 \mu \mathrm{m} \mathrm{Cu}$

Hohlraum Drive

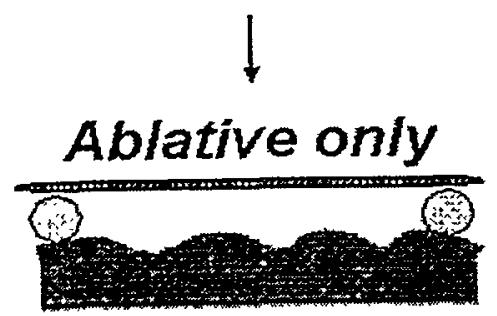

\section{Buffered + Ablative}

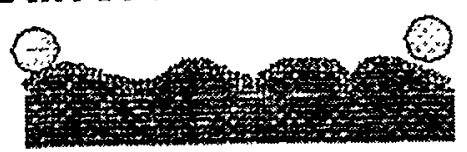

Fig. 1

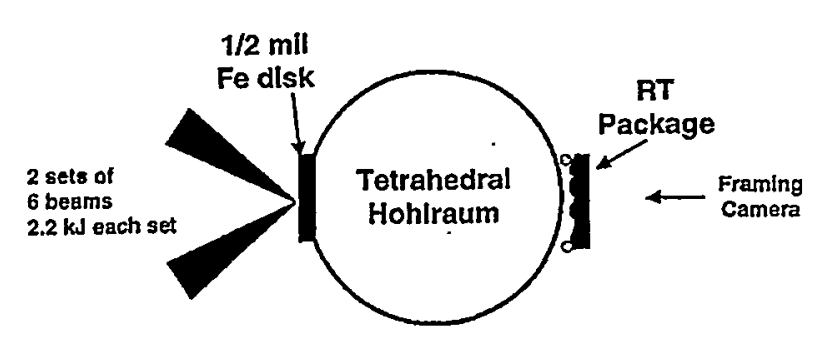

Fig.2 

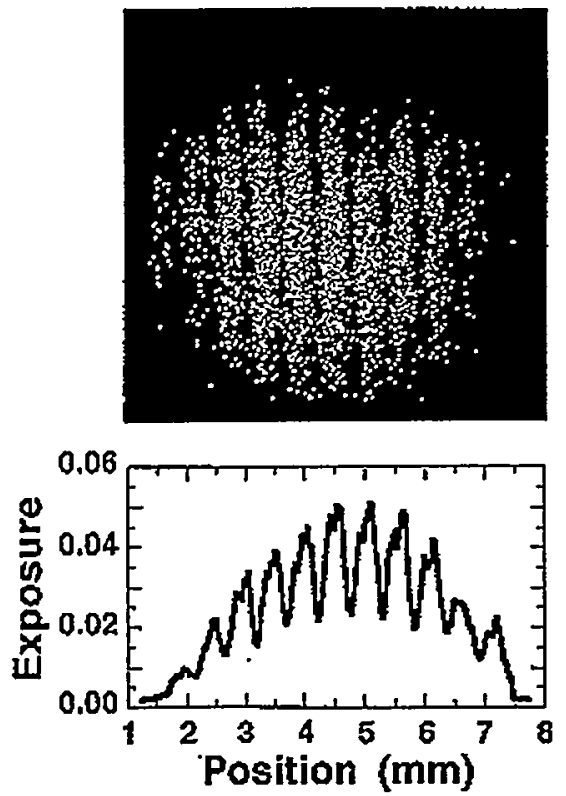

Fig.3
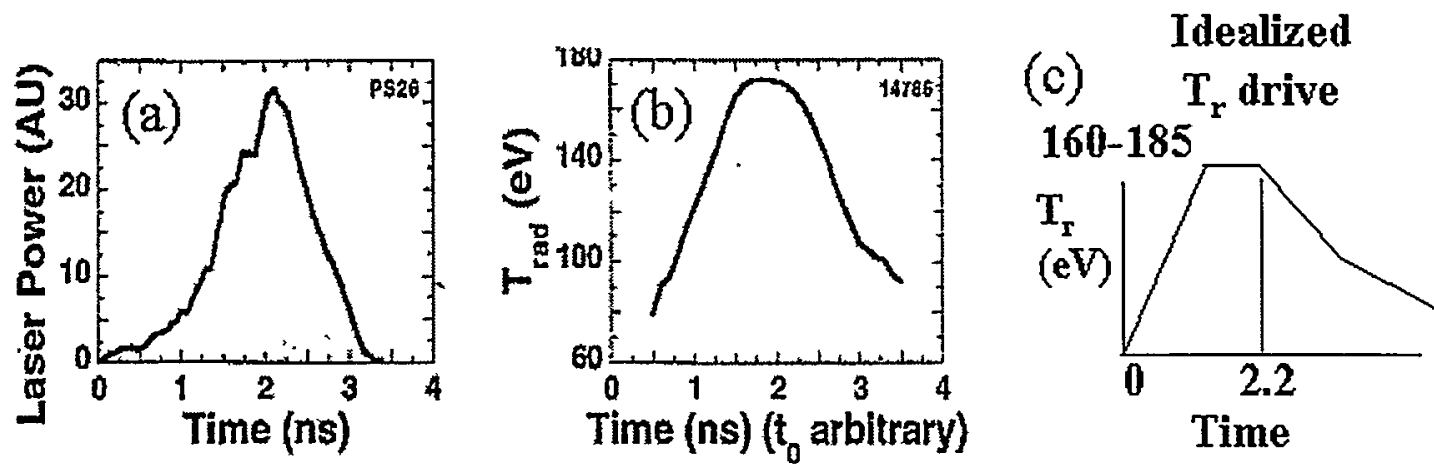

Fig. 4.
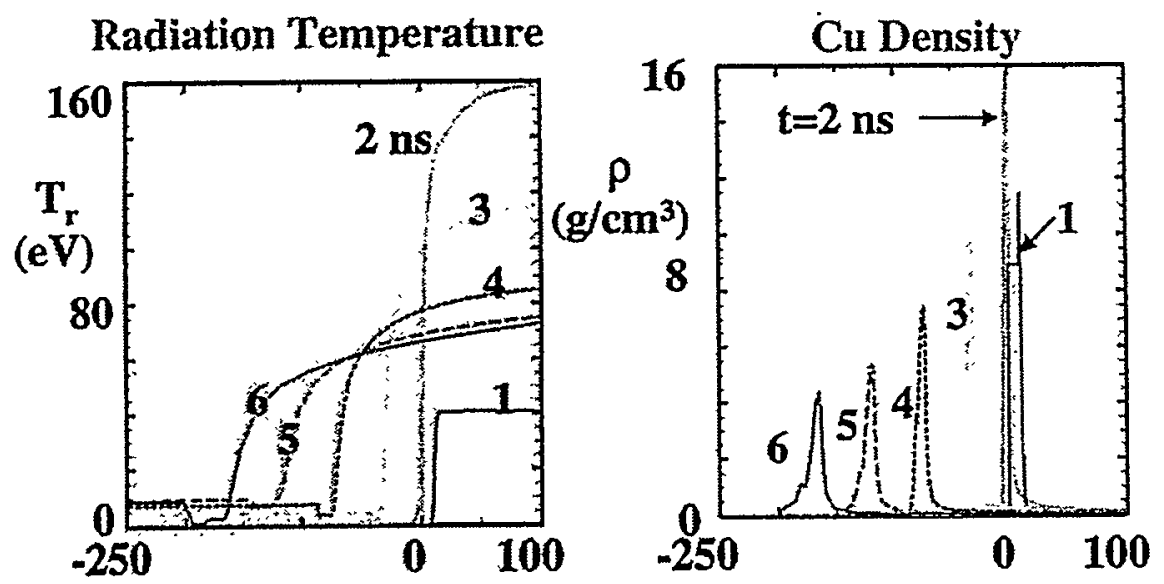

Fig. 5. 

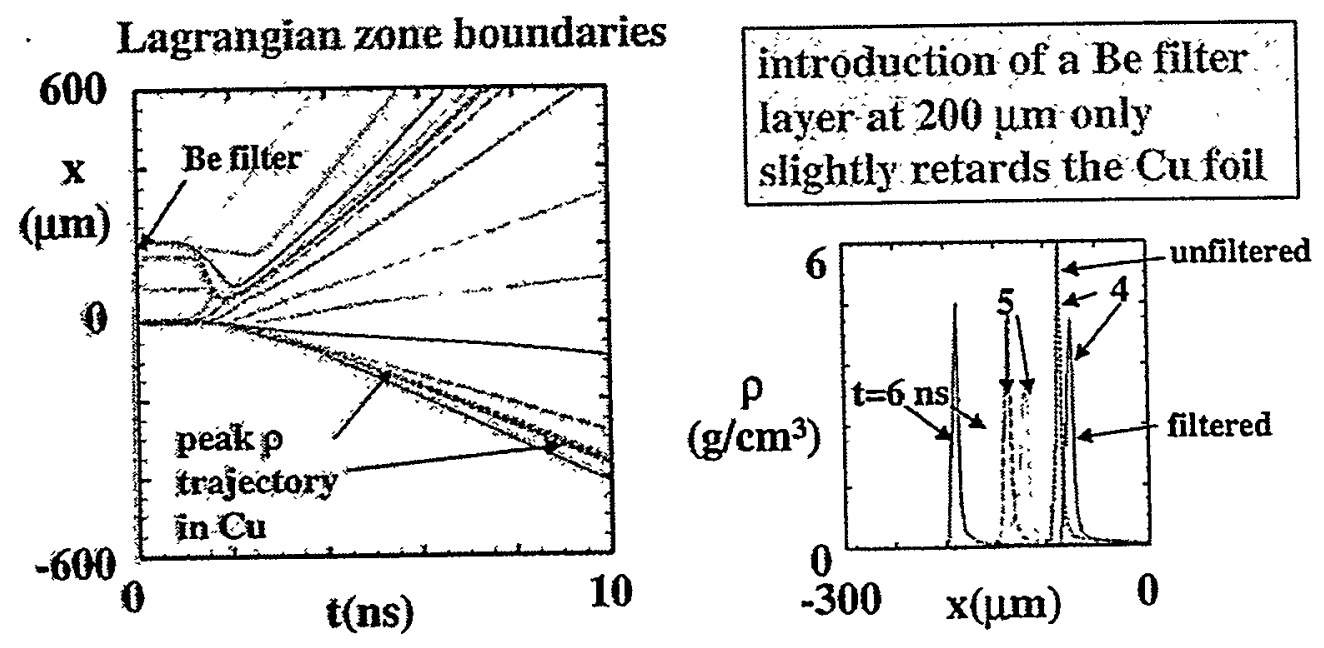

Fig. 6
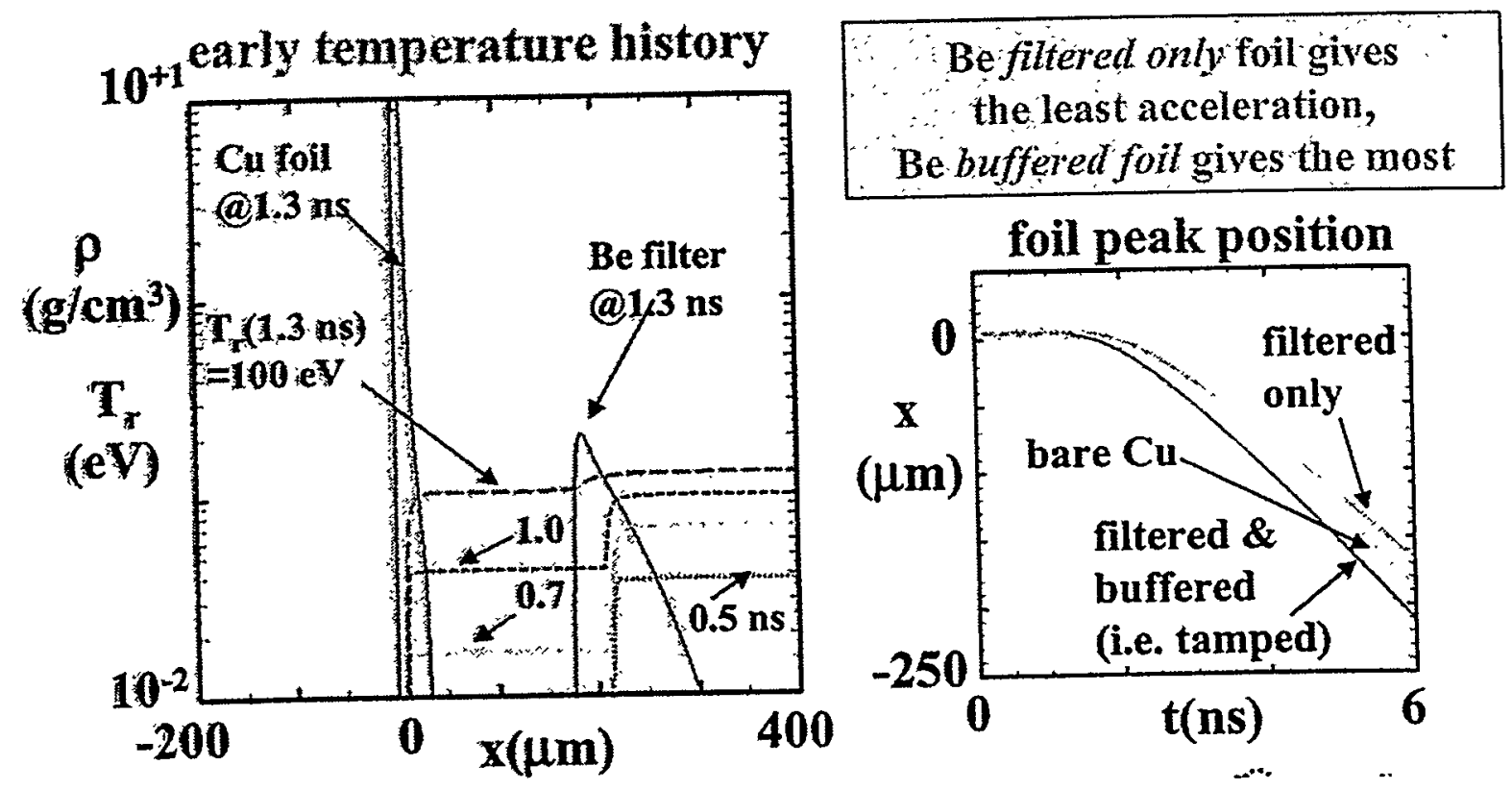

Fig. 7 


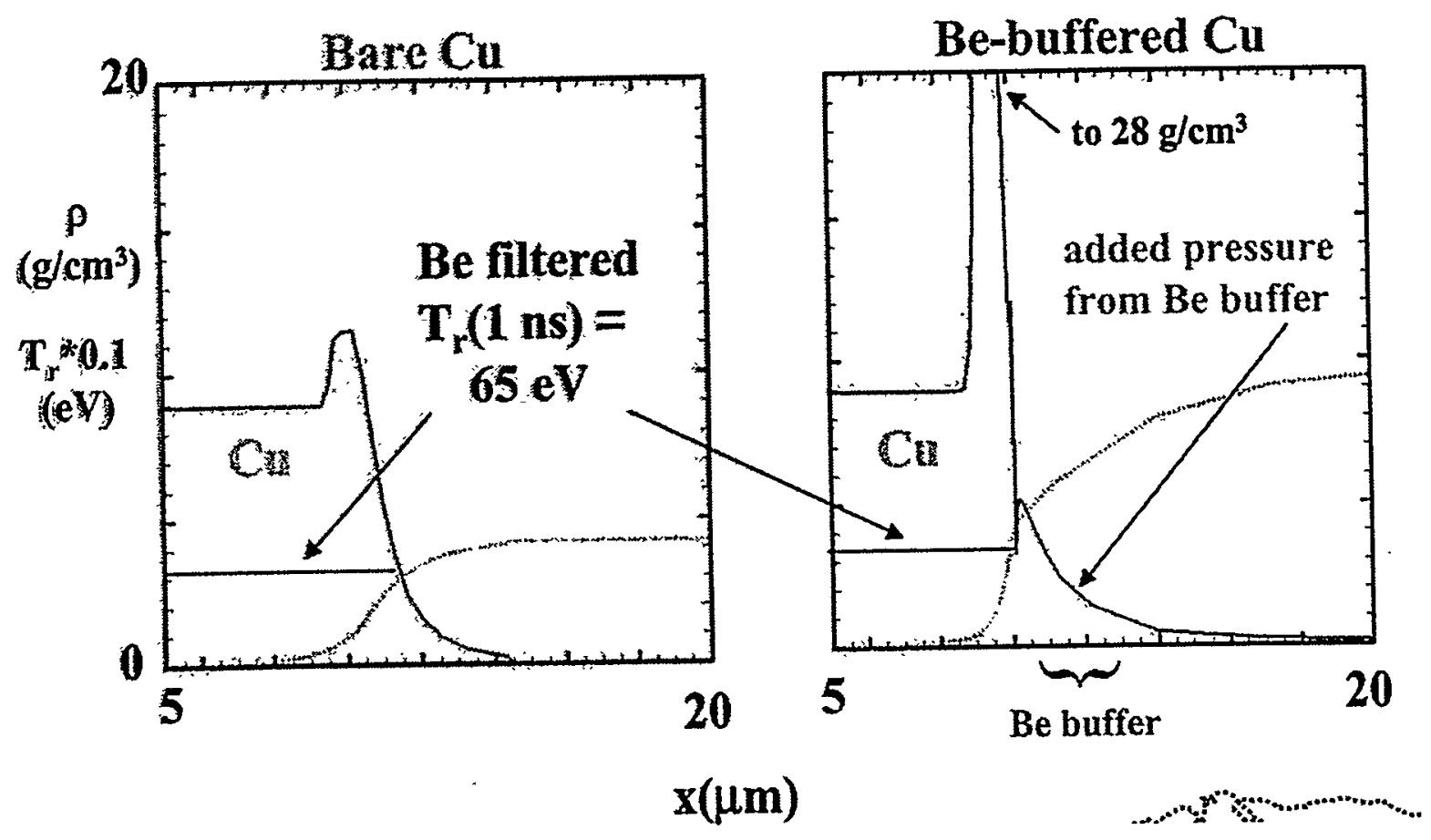

Fig. 8

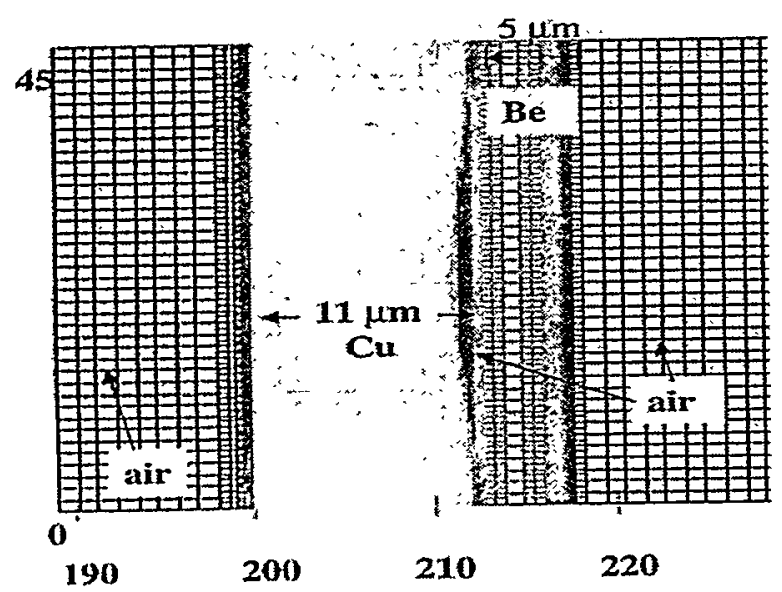

Fig. 9 

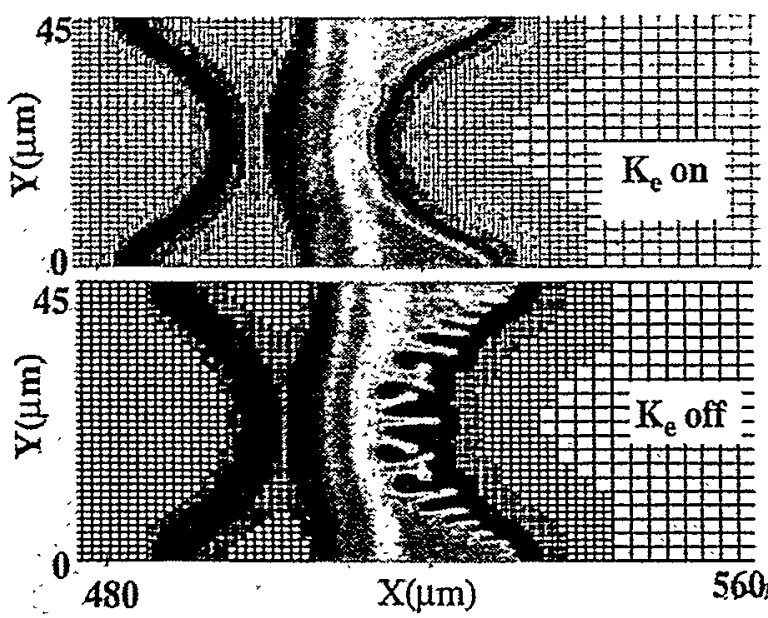

Fig. 10.

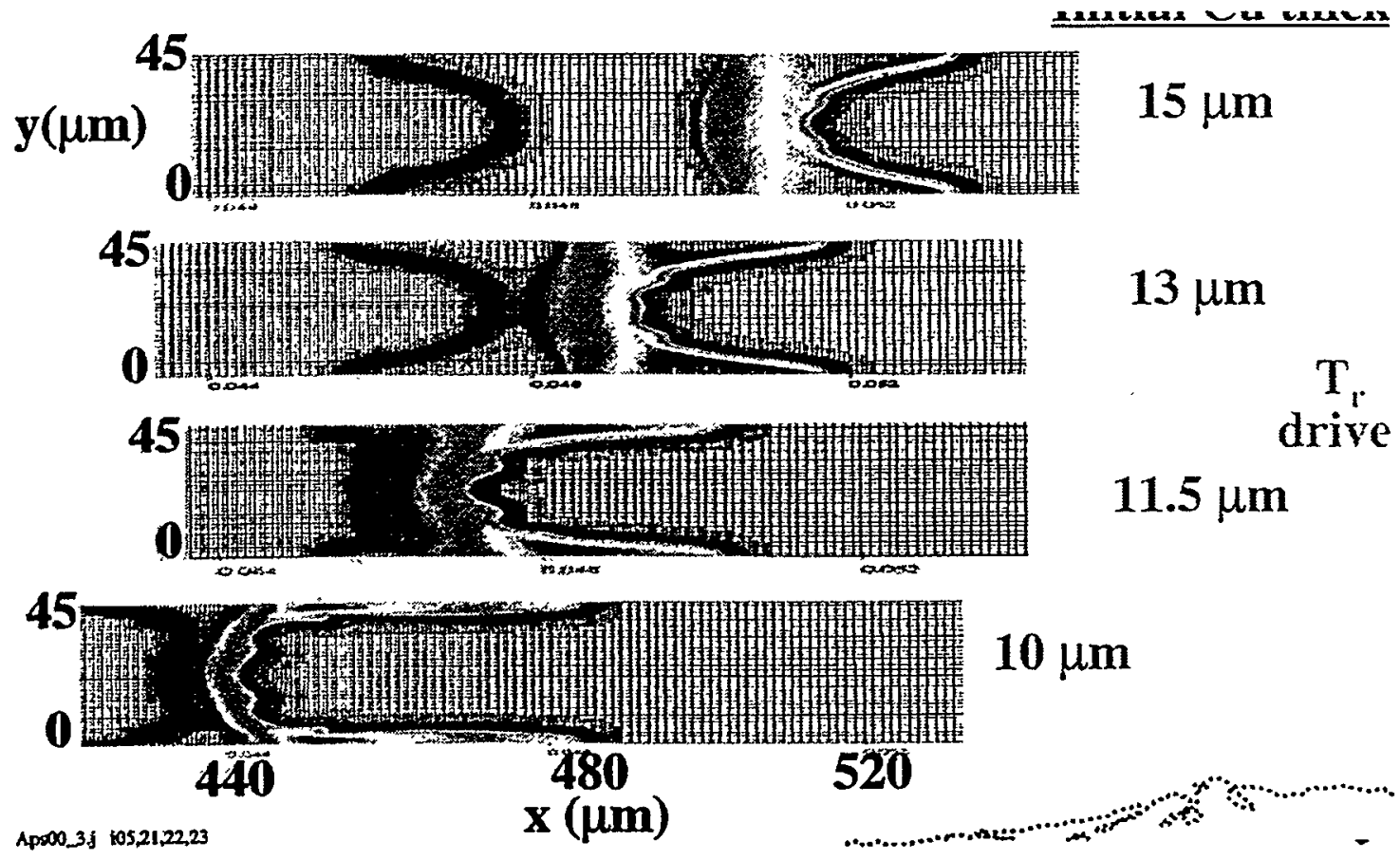

Fig. 11 


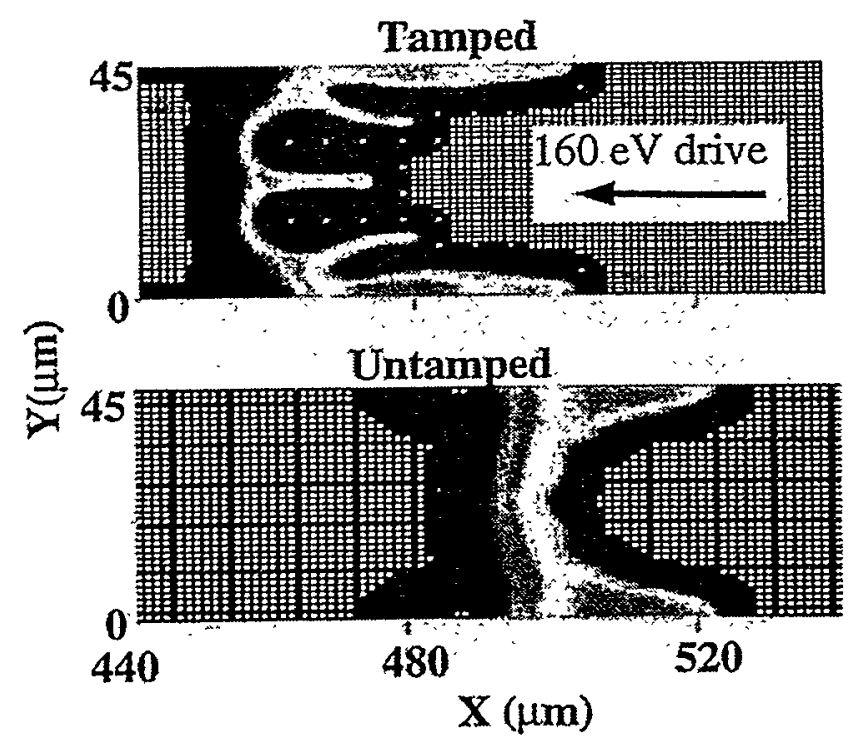

Fig. 12.
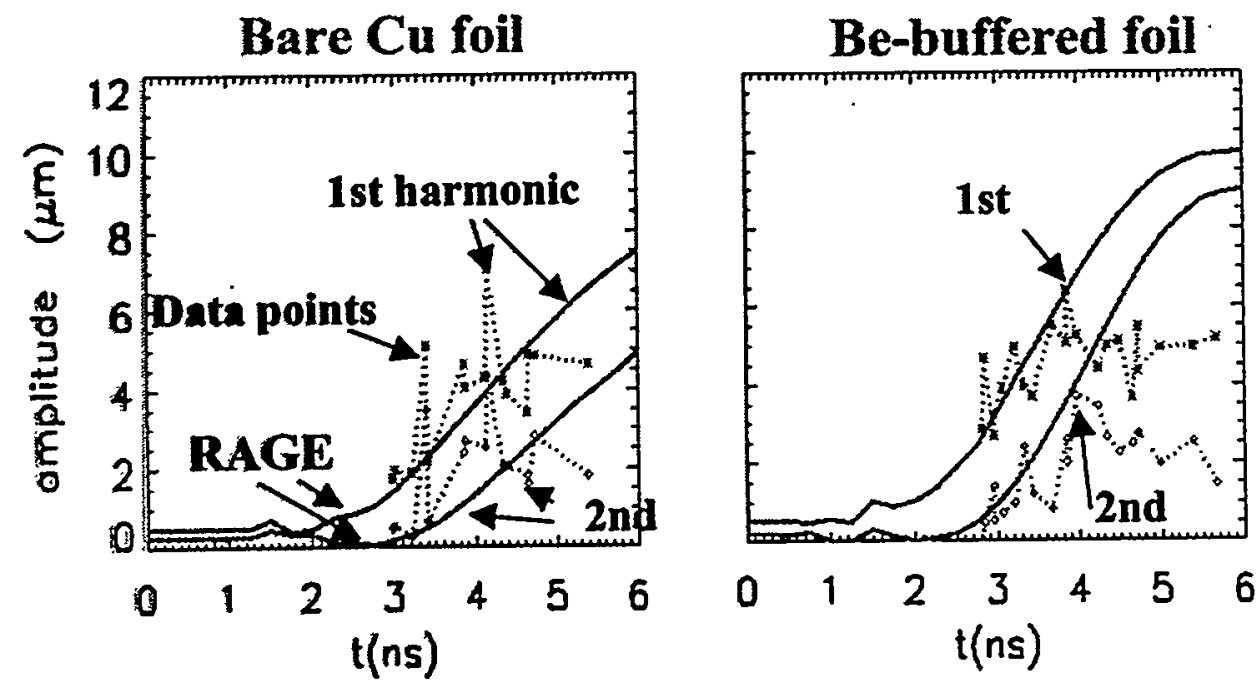

Fig. 13 


\section{Be-buffered $\mathrm{Cu}$ foil}

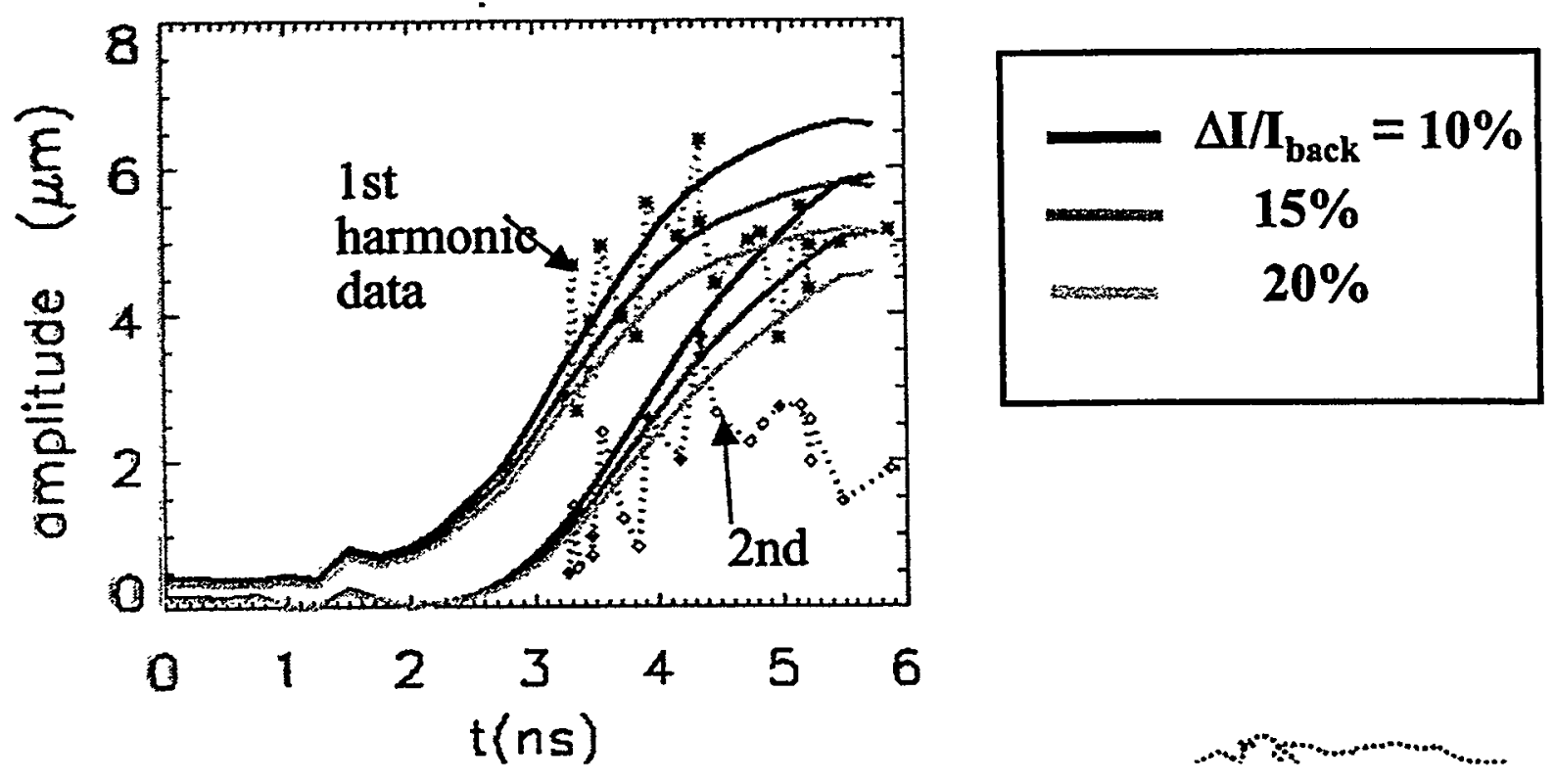

Fig. 14
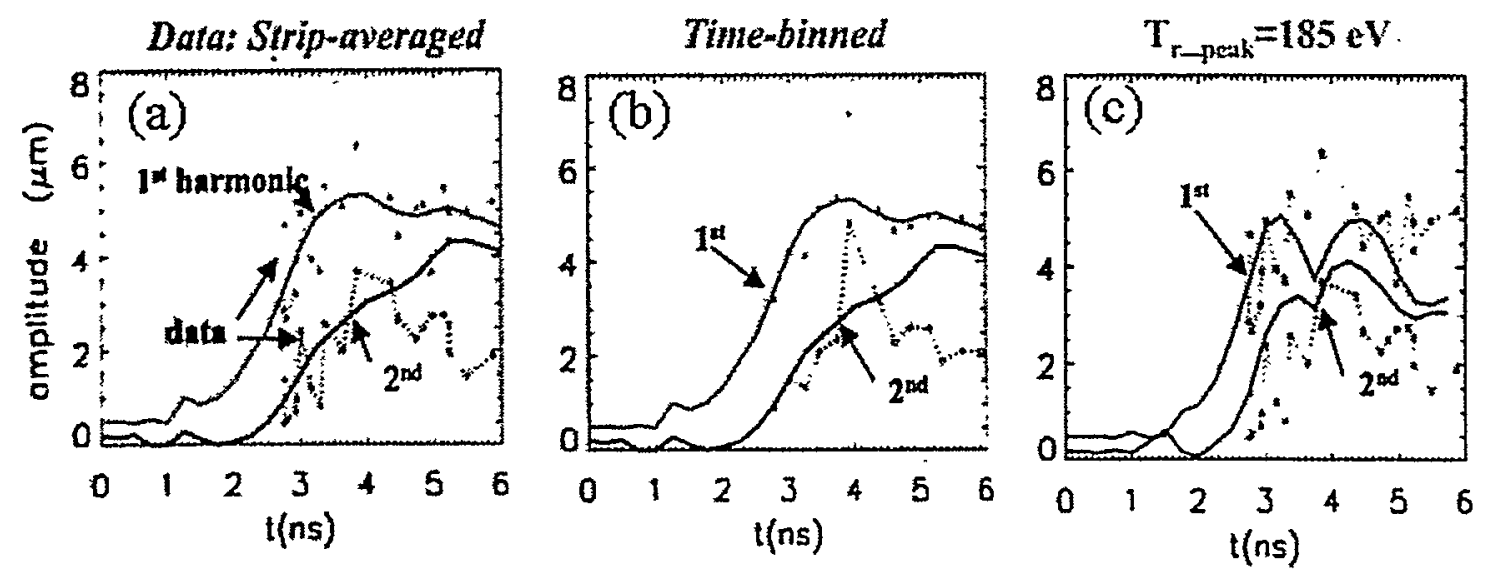

Fig. 15 